\title{
Prediction of Smear Positive TB Cases at Different Types of Designated Microscopy Centres, Karnataka, India
}

\section{Sharath Burugina Nagaraja ${ }^{*}$, Suresh Shastri², Jaya Prasad Tripathy ${ }^{3}$, Ghansham Sharma ${ }^{1}$, Shilpashree Madhav Kunjathur ${ }^{4}$, Anil Singarajipur ${ }^{2}$, Sarabjit Chadha ${ }^{3}$}

${ }^{1}$ Employees State Insurance Corporation Medical College and Post Graduate Institute of Medical Sciences and Research, Bangalore, India

${ }^{2}$ State TB Cell, RNTCP, Bangalore, India

${ }^{3}$ International Union against TB and Lung Diseases, New Delhi, India

${ }^{4}$ BGS Institute of Medical Sciences, Bangalore, India

Email: *sharathbn@yahoo.com

How to cite this paper: Nagaraja, S.B., Shastri, S., Tripathy, J.P., Sharma, G., Kunjathur, S.M., Singarajipur, A. and Chadha, S. (2017) Prediction of Smear Positive TB Cases at Different Types of Designated Microscopy Centres, Karnataka, India. Journal of Tuberculosis Research, 5, 258-264. https://doi.org/10.4236/jtr.2017.54027

Received: June 9, 2017

Accepted: November 20, 2017

Published: November 23, 2017

Copyright $\odot 2017$ by authors and Scientific Research Publishing Inc. This work is licensed under the Creative Commons Attribution International License (CC BY 4.0).

http://creativecommons.org/licenses/by/4.0/

\section{(c) (i) Open Access}

\begin{abstract}
Background: Under the Revised National Tuberculosis control Programme (RNTCP) in India, the designated microscopy centres (DMCs) form the basic unit of smear positive TB case detection in a district. There is a need by the programme managers to estimate the mean and range of smear positive tuberculosis (TB) cases that can be detected at DMCs located in different type of health facilities to channelize their resources. Methods: It is a cross-sectional study conducted in the state of Karnataka, India during January 2014 to December 2014 based on the compiled reports from past five years received from all the 30 districts of the state. The prediction was made based on the performance of these DMCs in the last five years using a modeling technique. Results: The proportions of the DMCs located at health facilities are Primary Health Institutions/Centres (PHIs)-73\%, Tuberculosis Units (TUs)-15\%, Medical colleges (MC) - 7\%, District TB centres (DTC) $-3 \%$ and Private Practitioners (PP) $-2 \%$. The maximum number of cases that can be detected at DTC is 3621 (SD 54), TU is 9224 (SD 90), PHI is 20,412 (SD 135), PP is 859 (SD 26) and MC is 8322 (SD 84). Conclusion: The predicted values will essentially serve as a tool for the programme managers of Karnataka to plan, strategize and monitor the performance of DMCs in the state.
\end{abstract}

\section{Keywords}

Normal Probability Model, Smear Positive TB, Prediction, India 


\section{Introduction}

In India, tuberculosis (TB) continues to be a major public health problem and it accounts for $25 \%$ of global burden. To contain the same, the Government of India is implementing the Revised National Tuberculosis Control Programme (RNTCP) since 1997 [1]. Under the programme, the main tool for diagnosis is examination of sputum by binocular microscopy at health centres called Designated Microscopy Centres (DMCs). In India, there are 13,000 DMCs located across the country and $\sim 1.7$ million presumptive $\mathrm{TB}$ cases are screened for $\mathrm{TB}$ disease and nearly 0.92 million are found to be sputum smear positive during 2015 [1]. The programme has moved towards universal access and there have been continuous efforts to screen and detect more TB cases.

The DMCs are spread across different type of health care facilities. Based on the population size, the programme has uniformly allotted a microscopy centre for every 0.1 million population at plain areas with an exception for tribal areas for which the population size is 0.05 million [1] [2]. The majority of the DMCs are located at hospitals in the general health system and few at private health facilities. Depending on the area of location the hospitals from general health system are classified as tertiary, secondary and primary health care hospitals/centres. By virtue of their location and specialist doctors availability the tertiary and secondary care hospitals attracts large number of patients; while, the DMCs at primary health Institutions/centre's (PHI) comparatively has less number of patients attending the hospital on outpatient basis; though, the number of DMCs situated at primary health centre is comparatively more than those located at tertiary care centres. The programme supports the DMCs by providing laboratory technicians (up to $20 \%$ - 50\%, depending on the state) and the necessary logistics for prompt conduct of sputum examinations.

Despite the constant efforts by the programme there has been no drastic change in the number of smear positive TB cases detected. The district programme managers are constantly involved in evolving out the strategies which could have impact on the district smear positive TB case detection rates. There is an inherent need for a tool by the programme managers to estimate the mean and range of smear positive TB cases that can be detected at DMCs located at different type of health facilities through which they can strategize their resources and prioritize their activities to achieve the programme objectives.

We conducted this study to predict the number of smear positive TB cases that can be detected at DMCs located in different type of health facilities based on the performance of these DMCs in the last five years using a modeling technique.

\section{Objectives}

Among the all smear positive TB cases detected at DMCs from Karnataka, 2009-13.

1) To determine the number (proportion) of smear positive TB cases detected 
at DMCs located in different type of health facilities.

2) To predict the number (quartile range) of smear positive TB cases that can be detected at DMCs from different health facilities.

\section{Methods}

It is a study conducted in the state of Karnataka during January 2014 to December 2014. The study involves the retrospective analysis of the records and reports received from the DMCs of 30 districts from the past five years. Under the programme, the monthly performance of DMCs which includes the numbers of presumptive $\mathrm{TB}$ cases screened and number found positive are received at district tuberculosis centre through a RNTCP reporting format called "Annexure M". The District submits the compiled report of all its DMCs to the state regularly and the state monitors the DMCs closely for presumptive TB case examination rates, slide positivity rates, results of on-site evaluation reports and random blinded re-checking reports consistently [2].

Routinely, the variables collected in the reporting formats includes number of presumptive tuberculosis cases examined and found positive for diagnosis, follow-up and repeat sputum examination. However, the type of DMC like Peripheral health institution, District TB centre, Tuberculosis unit, Medical College and private practitioner are not included in the routine reporting formats.

For the purpose of this study, we included all the DMCs from the years 2009-2013. Those DMCs which had complete data were analyzed and the total number of DMCs for the following years were 2009 (645), 2010 (630), 2011 (590), 2012 (563) and 2013 (645). The data on the type of health facility were collected from the districts based on their locations. They were classified as 1) District TB centres (DTC) 2) Medical Colleges (MC) 3) Peripheral health institution (PHI) 4) Tuberculosis unit (TU) 5) Private Practitioners (PP). Further, the DMCs were analyzed for number of presumptive TB cases examined for diagnosis and smear positive TB cases detected among those examined at all type of health facilities.

\section{Model for Prediction}

A model was constructed using normal probability distribution; we considered this model because the type of data which is dealt was discrete. As per Bernoulli trial, in a screening test, a smear positive case is considered as success while a negative case is considered as a failure. Hence, in order to predict the chances of occurrence of " $\mathrm{x}$ " number of positive cases, bernoulli probability law (Bernoulli distribution) is applicable [3]. In our study, the coverage for each of the centre is $1,00,000$ population and the number screened ranged from 8 to 9620 cases per year. Since, the population screened is a very large number, as per the law of large number (LLN) binomial distribution can be well approximated by normal distribution. In normal distribution the total number of smear positive cases detected will be considered as mean of binomial distribution. The normal distribu- 
tion model is a well accepted and validated model for prediction in the field of statistics. The normal distribution is applied assuming that the number of positive cases will remain almost consistent for at least next five years if all the conditions and resources remain the same in the coming years. The limitations for this prediction model are that it provides only the interval estimation which is based on five years data for the same type of centres.

\section{Calculation}

The normal probability law is calculated using the following formula [4].

$$
f(x ; \mu, \sigma)=P[X=x]=\frac{1}{\sigma \sqrt{2 \pi}} \mathrm{e}^{-\frac{(x-\mu)^{2}}{2 \sigma^{2}}}
$$

Considering, the smear positive $\mathrm{TB}$ case as a success and smear negative as a failure; the chances of occurrence of success has been estimated and prediction over number of positive cases has been made. The normal probabilities have been calculated using the following transformation: $Z=\frac{X-n p}{\sqrt{n p q}}$, where $Z$ is the standard normal variable having mean zero (0) and standard deviation unity (1), " $X$ " is the projected number of positive cases (number of successes) at which probability is to be calculated, $n p$ is the mean (actual number of positive cases) and $\sqrt{n p q}$ is the standard deviation of binomial distribution such that $p+q=1$.

\section{Results}

The smear positive TB cases detected at various types of health facilities are shown in Figure 1 as a function of total population screened. For all the type of health facilities there is a narrow confidence interval which explains that the fitted line is near to the linear data which is almost normal.

Of all the DMCs, the proportion of the DMCs located at health facilities like PHIs is $73 \%$, TUs is $15 \%$, Medical colleges is $7 \%$, District TB centres is $3 \%$ and private practitioners is $2 \%$. All the DMCs at health facilities has a progressive trend for smear positive TB cases while the peripheral health institution and medical college DMCs has steep rise in detection with increasing presumptive TB case detection. The best fit line has a narrow confidence interval which predicts the accuracy of the estimates.

Based on the model, the number of smear positive TB case detected at probabilities of $\mathrm{P}_{0}, \mathrm{P}_{25}, \mathrm{P}_{50}, \mathrm{P}_{75}, \mathrm{P}_{100}$ with mean and standard deviation at DMCs in various health facilities are shown in Table 1 . The probability $\mathrm{P}_{0}, \mathrm{P}_{25}, \mathrm{P}_{50}, \mathrm{P}_{75}, \mathrm{P}_{100}$ indicates the probability of detecting $25 \%, 50 \%, 75 \%$ and $100 \%$ of all the expected positive cases which is calculated cumulatively. As shown in Table 1, the maximum number $\left(\mathrm{P}_{100}\right)$ of cases (along with standard deviation-SD) that can be detected at a district through different type of health facilities is as follows: 1) DTC is 3621 (SD 54), 2) TU is 9224 (SD 90), 3) PHI is 20412 (SD 135), 4) PP is 859 (SD 26) and 5) MC is 8322 (SD 84). 

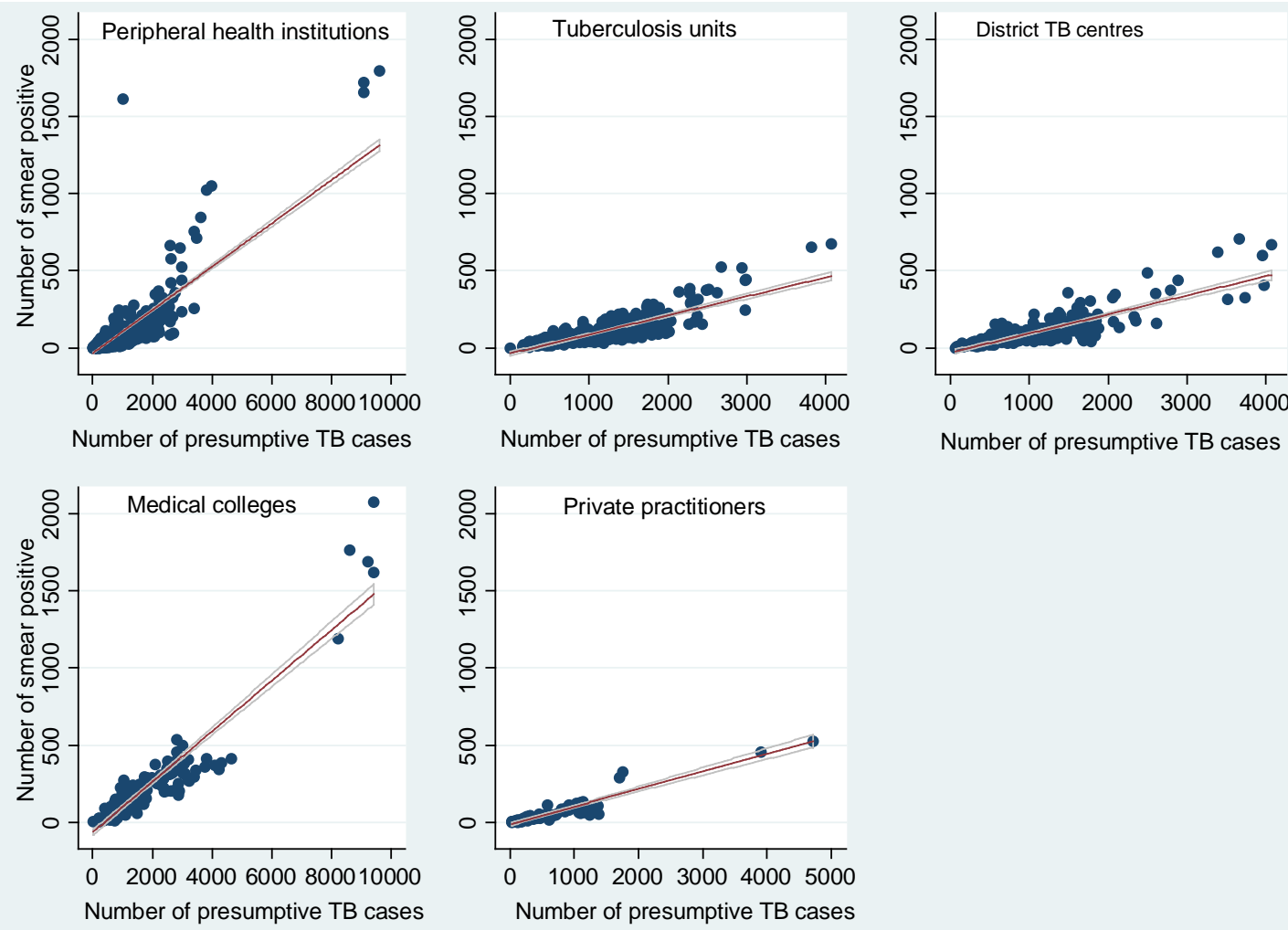

Figure 1. A scatter diagram showing the presumptive TB cases examined and smears positive TB cases detected at DMCs located in different type of health facilities, Karnataka, 2009-13.

Table 1. The number of smear positive TB case detected at probabilities of $\mathrm{P}_{0}, \mathrm{P}_{25}, \mathrm{P}_{50}, \mathrm{P}_{75}, \mathrm{P}_{100}$ with mean and standard deviation at DMCs in various health facilities.

\begin{tabular}{cccccccc}
\hline $\begin{array}{c}\text { Types of } \\
\text { Health } \\
\text { facilities }\end{array}$ & $\mathrm{P}_{0}(\mathbf{0} \%)$ & $\mathrm{P}_{25}(\mathbf{2 5 \% )}$ & $\mathrm{P}_{50}(50 \%)$ & $\mathrm{P}_{75}(\mathbf{7 5 \% )}$ & $\mathrm{P}_{100}(100 \%)$ & $\mathrm{P}($ Mean $)$ & $S D$ \\
\hline DTC & 3200 & 3370 & 3407 & 3444 & 3621 & 3407.0 & 54.78 \\
TU & 8523 & 8813 & 8873 & 8935 & 9224 & 8873.4 & 90.10 \\
PHI & 19,519 & 19,874 & 19,966 & 20,057 & 20,412 & $19,965.2$ & 135.69 \\
PP & 655 & 739 & 757 & 775 & 859 & 756.8 & 26.24 \\
MC & 7669 & 7939 & 7995 & 8052 & 8322 & 7995.0 & 83.97 \\
\hline
\end{tabular}

\section{Discussion}

This is one of the first studies that predict the number of sputum smear positive TB cases detected at DMCs located in different health facilities of Karnataka based on normal probability distribution model. Accordingly, the major contributions for a smear positive TB case detection rate in a district from the DMCs are located at PHI, TU, MC, DTC and PP. The trend for the next few years might be almost consistent as the data used for extrapolation of the estimates is based on the programme data from the past five years. However, it is most unlikely to expect an increasing trend line in smear positive case detection rate until and 
unless there is major strategic changes in policy and implementation. The study findings have following programmatic implications.

First, the large numbers of cases are detected from the DMCs located at the PHIs, TUs and Medical colleges. The district programme managers to achieve the goal of 180 presumptive TB cases examination per lakh population with $10 \%$ $15 \%$ slide positivity rates in the districts should concentrate their efforts on strengthening the PHI and TU DMCs. The programme managers can use the prediction values provided in the table below to calculate their performance against the predicted value for the state.

Second, at the local level the district programme managers need to develop various strategies to improve the DMCs performance. One such strategy for a DMC is "feeder" strategy which helps in ensuring all those presumptive TB chests symptomatic from other feeding PHCs are routed to the DMC without missing any of the referred cases. The information technology (IT) enabled systems to monitor the human resources, the logistics and the patient flow from the feeder PHCs to DMCs will possibly increase the quality of supervision and monitoring which will have direct effect on case detection rate. Inadvertently, the general health system strengthening has to happen in terms of quality of health services provided at the PHCs which will draw all sections of people for health services utilization. The programme needs to customize its advocacy, communication and social mobilization activities based on the DMC laboratory data while it should also conduct surveys that could gather information from the communities' perspective to increase the utilization of laboratory services.

Third, introduction of available newer technologies like fluorescent microscopes at PHCs level and cartridge based nucleic acid amplification test at tuberculosis units will help in increasing the yield of smear positive TB cases. However, to garner the evidence the programme may conduct feasibility and cost effectiveness studies before proposing a change in a policy.

To accomplish the targets as mentioned by the "End TB strategy", the programme certainly has to take bold steps [5]. Inevitably, the case detection remains the priority for the years to come. The predicted values will essentially serve as a tool for the programme managers of Karnataka to plan, strategize and monitor the performance of DMCs.

\section{Conflicts of Interest Statement}

We declare that we have no conflicts of interest.

\section{Contributorship Statement}

The idea was conceived by SBN, SS, GS, JPT, SMK, AS and SC. The authors have contributed in writing and approving the final manuscript.

\section{References}

[1] Ministry of Health and Family Welfare (MoHFW) (2014) Central TB Division, Annual Status Report-TB India. 
[2] Central TB Division, Ministry of Health and Family Welfare (2005) Revised National Tuberculosis Control Programme Laboratory Network Guidelines for Quality Assurance of Smear Microscopy for Diagnosing Tuberculosis.

[3] Gupta, S.C. and Kapoor, V.K. (2003) Special Discrete Probability Distributions. Fundamental of Mathematics. June 2002. Sultan Chand and Sons, New Delhi, 8.4-8.27.

[4] Chand, S.C. and Kapoor, V.K. (2003) Special Continuous Probability Distribution. Fundamental of Mathematics. 2002nd Edition, SC Chand and Sons, New Delhi, 9.4-9.5.

[5] World Health Organization (WHO) (2015) WHO End TB Strategy. WHO. http://www.who.int/tb/post2015_strategy/en/ 